\title{
LA RESPONSABILIDAD SOCIAL EMPRESARIAL, SU RELACIÓN CON LA GESTIÓN DEL CONOCIMIENTO Y EL CAPITAL INTELECTUAL
}

\section{CORPORATE SOCIAL RESPONSIBILITY ITS RELATIONSHIP WITH THE MANAGEMENT OF KNOWLEDGE AND INTELLECTUAL CAPITAL}

\author{
Avellán Herrera Nilda Alexandral
}

\begin{abstract}
Resumen
En el ámbito empresarial la creación de valor a través de una adecuada gestión del conocimiento (GC) se ha vuelto cada vez más necesaria para la sobrevivencia de las empresas; ello, en un mercado que cada día demanda estar a la vanguardia de la globalización de la información y su cambio vertiginoso. Asimismo, se habla de los activos intangibles como el capital intelectual (Cl), que, mediante sus componentes, contribuye a la creación de valor agregado para una empresa con respecto a la competencia. Por otra parte, la responsabilidad social empresarial (RSE) ayuda a complementar muy bien a las otras variables, como una fuente de ventaja competitiva empresarial, y, al mismo tiempo, favorece el desarrollo sostenible. En el presente artículo se abordan los conceptos antes señalados desde un nivel de investigación descriptivo mediante una revisión bibliográfica de investigaciones anteriores principalmente de artículos científicos de actualidad referentes al tema, teniendo como principal objetivo analizar la relación que existe entre la RSE, la GC y el Cl en las organizaciones, obteniendo como principal conclusión que un modelo de gestión del conocimiento basado en valores morales y éticos, así como en preceptos de responsabilidad social, permitirá a las empresas lograr valor agregado que permita diferenciarse de la competencia.
\end{abstract}

\section{Palabras clave}

Gestión del conocimiento, capital intelectual, responsabilidad social empresarial, contabilidad social, activos intangibles.

Clasificación JEL: M13, M14.

\begin{abstract}
In the business world, the creation of value through an adequate knowledge management (KM) has become increasingly necessary for the survival of companies in a market that every day demands to be at the forefront of the globalization of information and its vertiginous change. Likewise, we talk about intangible assets such as intellectual capital (Cl), which through its components allows creating added value for a company against its competitors. On the other hand, corporate social responsibility (CSR) allows to complement the other variables very well as a source of business competitive advantage and, at the same time, contributes to sustainable development. In this paper, the concepts mentioned above are addressed from a descriptive research level through a bibliographic review of previous investigations mainly of current scientific articles on the subject, aiming to analyze the existing relationship between CSR, CG and the Cl in organizations, concluding that a knowledge management model based on moral and ethical values, as well as on social responsibility precepts will enable companies to achieve additional value to differentiate themselves from the competition.
\end{abstract}

\section{Keywords}

Knowledge management, intellectual capital, corporate social responsibility, social accounting, intangible assets.

JEL Classification: MI3, MI4. 


\section{Introducción}

"El entorno empresarial actual, caracterizado por la incertidumbre, el cambio constante y la complejidad, confiere un protagonismo especial al conocimiento, un elemento esencial para el desarrollo organizacional al constituirse en su mayor activo y mayor fuente de riqueza" (Angulo, 2017: 55).

El conocimiento se convierte, así, en un generador de valor y ventaja competitiva para las empresas que buscan obtener beneficios en diferentes ámbitos no solo en el aspecto económico, sino en lo social y ambiental. Se entiende entonces que la gestión del conocimiento es "el conjunto de procesos que utilizan el conocimiento para la identificación y explotación de los recursos intangibles existentes en la empresa, así como para la generación de otros nuevos" (Ortiz de Urbina Criado, 2003: 285).

De ahí que si la gestión del conocimiento (GC) ayuda a reconocer aquellos activos intangibles con los que cuenta una empresa, no se puede dejar de lado al capital intelectual (Cl); esto, principalmente, porque "los activos intangibles tienen su origen en los conocimientos, habilidades, valores y actitudes de las personas. A estos activos intangibles se les denomina capital intelectual" (Madrigal, 2009: 76).

Como ya se dijo, en la actualidad las organizaciones aspiran a crear valor a través del conocimiento que poseen y tratan de compartirlo, ya sea en forma interna o externa, ya sea con sus grupos de interés, entre los que se encuentran los empleados, gerentes, propietarios, proveedores, sociedad, Gobierno, acreedores y clientes. Es así como hoy en día está cobrando mucho auge el asunto de ser una empresa socialmente responsable mediante programas orientados hacia la contribución en lo ambiental, social y económico. Dichos programas, a su vez, aportan a cada uno de los componentes del capital intelectual, tomando en cuenta que ambos conceptos son fuente de ventaja competitiva en un entorno económico y social en donde los intangibles asumen un papel protagonista en la llamada economía de la información.

La consideración de la RSC y el Cl en las organizaciones son obligadas [sic] en los momentos actuales, dada su trascendencia en la obtención de ventajas competitivas. Nos referimos a estrategias capaces de participar en la generación de rendimientos crecientes y en la obtención de mejores resultados. (Castilla-Polo, Gallardo-Vázquez, 2015: 14)

Por consiguiente, ser socialmente responsable contribuye a su vez al $\mathrm{Cl}$ ya que, si se considera la inversión en programas de RSE que generan nuevos conocimientos y habilidades, ambos contribuirían al capital humano de una empresa; de igual manera, la inversión en tecnología, patentes, marcas, propiedad intelectual, cultura organizacional, bases de datos entre otros estarían directamente relacionados con el capital estructural, y la reputación, imagen de marca, cartera de clientes, alianzas contribuye al capital relacional que son las relaciones con sus clientes externos.

[...] ser socialmente responsable significa invertir más en su $\mathrm{Cl}$, ya que ayuda a gestionar el conocimiento relevante para la organización y que, en línea con los objetivos de la misma, contribuye a generar valor a nivel interno y externo; esto es, pensando no sólo en el accionista o propietario, sino también en el empleado, cliente y la sociedad en general. (Vaca, Moreno, Riquel, 2007: 31-36)

Ahora bien, es de suma relevancia que las organizaciones dispongan de las herramientas correctas para medir los resultados de la gestión que se haga; sin embargo, muchas "adolecen [...] de técnicas para su ade- 
cuada gestión; no tienen métodos ni herramientas para su medición; no existen análisis sobre la capacidad innovadora de las organizaciones" (Ruiz, Font, Lazcano, 2016: 121).

Por ende, para los profesionales del área contable es un desafío bastante importante la medición y evaluación de los activos intangibles. Para ello deberían contar con el apoyo de la gerencia y tener el soporte tecnológico que les permita, además, elaborar los reportes tradicionales, recolectar, procesar y obtener la información para una adecuada gestión de los intangibles.

Por no encontrarse reflejado el capital intelectual (capital humano, estructural y relacional) en los estados financieros de las empresas, existe un desconocimiento de la importancia y valuación de éstos activos intangibles por parte de los administradores y cómo influyen en la rentabilidad financiera de una organización. (Pardo et al., 2017: 203)

En efecto, la contabilidad ha tenido que evolucionar junto con las necesidades que exige el entorno actual con nuevos in-

\section{Marco teórico}

\section{Gestión del conocimiento}

La gestión del conocimiento le permite a una organización crear procesos más cónsonos para que aquellas competencias excepcionales del talento humano sean recabadas, procesadas, almacenadas y transmitidas de la manera más adecuada. Ello se convierte en una ventaja competitiva frente a empresas del mismo sector con productos o servicios que favorezcan la obtención de ingresos o beneficios sostenibles en el tiempo.

La gestión del conocimiento significa formalizar procesos de creación, desarrollo, difusión y explotación del conocimiento, dando lugar a la generación de valor agregado a los productos y servicios que ofer- formes que revelen aquellos intangibles que por mucho tiempo habían estado inadvertidos en las empresas. Entre estos nuevos reportes se pueden mencionar el informe de capital intelectual, en el que se presentan indicadores para cada uno de sus componentes: humano, estructural y relacional. Asimismo, también están los informes de sostenibilidad, que incluyen algunos estándares con el fin de medir y evaluar el impacto de los programas implementados. Dichos informes se analizan en los apartados "marco teórico" y "resultados" de este artículo.

En definitiva, las empresas que obtengan, generen, procesen y transmitan el conocimiento de manera adecuada tienen una ventaja competitiva frente a las que no, lo que del mismo modo está cambiando la perspectiva de la contabilidad. Esta última es vista no solo desde una dimensión económica, sino también social, resultado que se convierten en un activo de naturaleza intangible que aportan al capital intelectual importante en el desarrollo económico y social de las empresas.

tan las organizaciones, lo que incide en el incremento de sus ingresos y beneficios por medio de la innovación de la oferta, la reducción de costos, (y) ganando de esta manera capacidad competitiva. (Solíz, Mena, Lara, 2017: 72)

Así, a través de la gestión del conocimiento se origina un valor agregado que representa beneficios para la competitividad empresarial y la cultura organizacional, con el objeto de alcanzar los réditos económicos esperados, pero sin dejar de lado la importancia de retribuir a la sociedad y al medioambiente lo que ambas variables entregan a las empresas siendo socialmente responsables. 


\section{Responsabilidad social empresarial}

En respuesta a la nueva tendencia mundial, orientada hacia el desarrollo sostenible, surge la responsabilidad social empresarial (RSE). Dicho modelo de gestión empresarial se fundamenta en tres pilares principales: responsabilidad económica, responsabilidad social y responsabilidad ambiental. Estos ejes son esenciales para las organizaciones, que ven en la inversión socialmente responsable una oportunidad de contribuir con programas, planes o proyectos que busquen una mejor solución a tantos problemas que aquejan a la sociedad y al medioambiente; y que, a su vez, obtengan réditos económicos por esta gestión.

La RSE integra un conjunto de prácticas, estrategias y sistemas de gestión que persiguen un nuevo equilibrio entre las dimensiones económica, social y ambiental. Se busca conciliar eficacia y eficiencia empresarial con principios sociales de democracia, autoayuda, apoyo a la comunidad y justicia distributiva, sumando la aplicación de una nueva valoración, la efectividad, el impacto a terceros. (Caridad, Hernández, Pelekais, 2014: 41)

De acuerdo con lo expuesto, la RSE implica ir más allá del simple hecho de ayudas sociales: requiere que la organización ejerza un comportamiento ético con todos sus grupos de interés, respete las normativas locales e internacionales en la búsqueda de contribuir a los objetivos globales de desarrollo sostenible, ser transparentes en las decisiones que puedan afectar a la sociedad y al

\section{Capital intelectual}

"El capital intelectual que ha sido definido como el conjunto de recursos y capacidades de una organización que, pese a no estar reflejados en los estados contables tradicionales, generan o generarán valor para la organización en el futuro" (Cegarra, medioambiente. Sobre todo rendir cuentas por las decisiones que conlleven tanto aspectos negativos, en perjuicio de la sociedad y el ecosistema, como por aquellas que signifiquen mitigar ese impacto negativo a través del mejoramiento de la calidad de vida de empleados, comunidad. En especial, se aspira a que favorezca la disminución del impacto ambiental que actualmente vive el planeta.

Barabraham (2017), quien cita a Fernández Lorenzo y a Geba (2005), menciona:

La Contabilidad Social se identifica como un conjunto de conocimientos, elementos y procesos de la disciplina contable que, con un enfoque socioeconómico, permite saber, de manera mayoritariamente cuantificada, los impactos sociales producidos por los entes tanto en el medio ambiente natural como en el cultural, y es útil para conocer el cumplimiento de la Responsabilidad Social asumida por las organizaciones. (p. 8)

Por consiguiente, si hablamos de que los programas de responsabilidad social empresarial influyen en el recurso humano, entonces es parte de la contabilidad social medir el efecto de los programas implementados en la generación de nuevos conocimientos, habilidades, competencias, valores, que mediante una adecuada gestión y procesamiento de los resultados, puedan traducirse en un valor agregado para las organizaciones. Para esto, algunos autores también hablan de un reporte llamado el "estado del capital intelectual" o The Intangible Global Report.

Martínez, 2017: 169). En décadas pasadas se pensaba que los activos tangibles de una empresa eran los que aportaban mayor valor a la organización; sin embargo, este concepto quedó atrás con la aparición de los llamados activos intangibles. 
También son activos intangibles las capacidades que se generan en la organización cuando los recursos empiezan a trabajar en grupo; muchos, en lugar de capacidades, hablan de procesos o rutinas organizativas. Toda empresa debe desarrollar o adaptar la colección de indicadores que mejor pueda reflejar el valor de su Capital Intelectual y tomar sus decisiones teniendo en cuenta ese valor. (Bravo, Alfonzo, 2015: 229)

Es así como en el ámbito empresarial se dieron cuenta de que la creatividad, la innovación, los valores, las habilidades, el co- nocimiento y las capacidades de su talento humano dan pie a información intangible muy valiosa para la organización que, a pesar de no constar en los informes contables que se presentan a la gerencia, son de mucha valía, dado que crean valor a futuro, al que llamaron capital intelectual. Se trata, pues, de un activo intangible que permite crear valor intrínseco para la organización y una ventaja competitiva frente a otras empresas de la misma industria o sector.

Entre los componentes del capital intelectual se pueden mencionar:

Figura 1. Componentes del capital intelectual

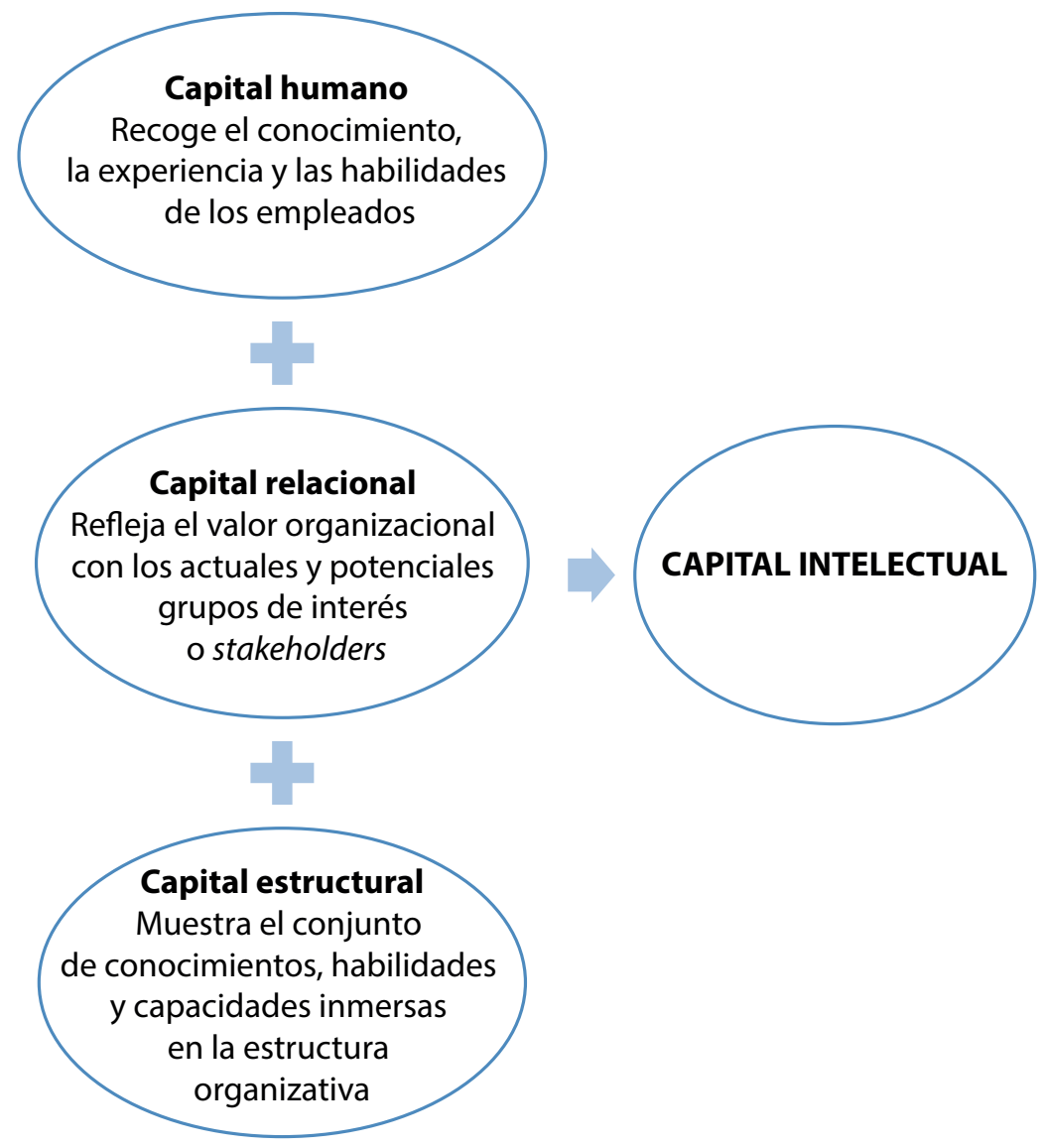

Elaboración propia a partir de Castilla-Polo, Gallardo-Vázquez (2015)

Los componentes del capital intelectual son, en su mayoría, de naturaleza subjetiva; por ende, gran parte de las empresas y sus administradores le restan importancia a su medición y evaluación. Así, se desconoce la incidencia de estos activos intangibles 
en la rentabilidad de las empresas, además de no reflejarse el capital intelectual en sus

\section{Metodología}

El nivel de investigación que se expone en este trabajo es de carácter descriptivo, en el que se destacan, definen y analizan las características más distintivas de cada uno de los términos que delimitan el tema del artículo. Por otra parte, el estudio comprendió un tipo de investigación bibliográfica a través de revisiones de indole documental sobre las variables de estudio, con el objetivo de analizar la relación entre la responsabilidad social empresarial, la gestión del conocimiento y el capital intelectual en las organizaciones. Las fases que se cum- estados financieros (Pardo, Armas, Chamba, 2017: 203). plieron son las siguientes: revisión teórica de los principales términos de estudio mediante bases de datos científicas. Para el análisis de los resultados y discusión, se consideraron las principales tendencias respecto a las diferentes clases de informes con el fin de reportar los intangibles de una empresa. Para finalizar, se presentan las conclusiones del material recopilado y analizado que ayuden a despertar el interés en el tema, así como también ser fuente de consulta para futuras investigaciones con respecto al mismo asunto.

\section{Resultados y discusión}

\section{Los informes de sostenibilidad y de capital intelectual}

Si bien es cierto que en la mayoría de los países de Latinoamérica no existe una legislación ni una forma estandarizada para exponer los resultados de los programas de RSE, la gran mayoría de las empresas optan por buscar la mejor manera de mostrárselos a sus stakeholders a través de los llamados informes de sostenibilidad. Para ello existen algunas guías; por ejemplo:

Figura 2 Principales guías para la elaboración de informes de sostenibilidad

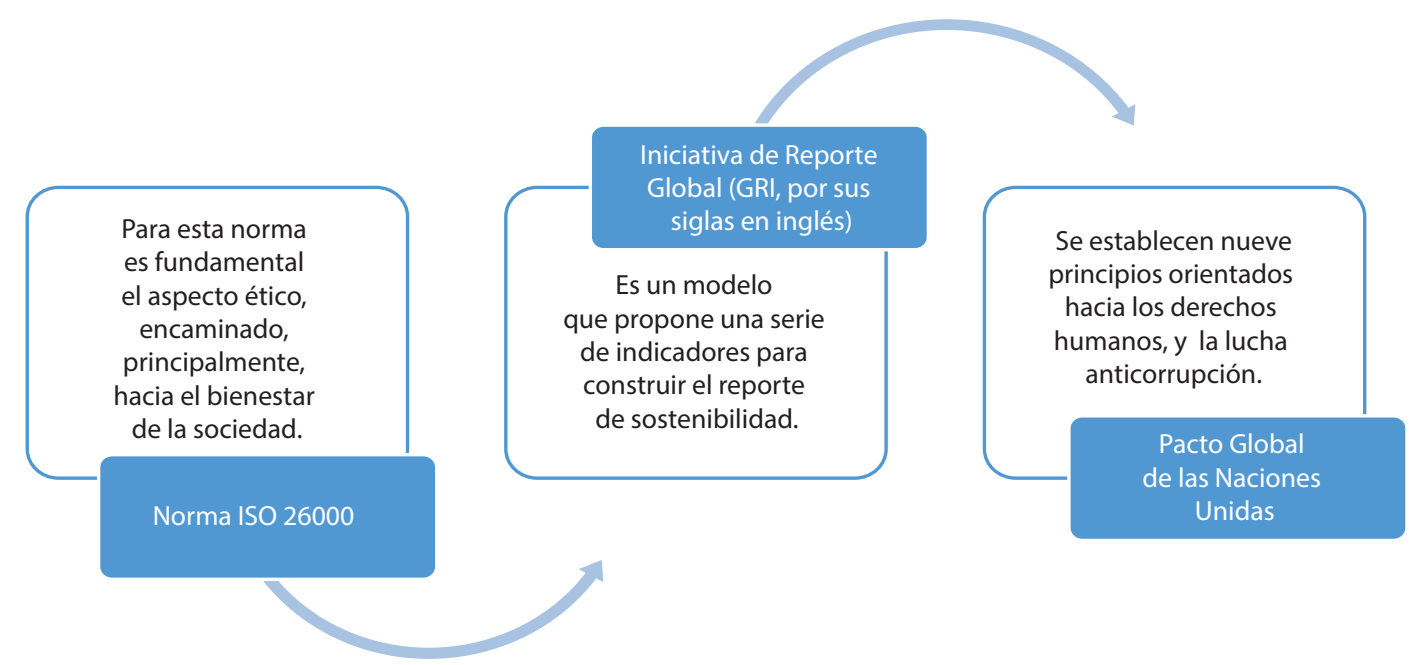

Elaboración propia 
De las guías mencionadas en la ilustración 2 se destaca la Iniciativa de Reporte Global, (GRI, por sus siglas en inglés), la cual establece algunos estándares bases para la elaboración de los informes de sostenibilidad, que siguen a continuación:

Tabla 1. Estándares GRI para la elaboración de informes de sostenibilidad

\begin{tabular}{|c|c|}
\hline \multirow{6}{*}{$\begin{array}{l}\text { GRI 200: ESTÁNDARES TEMÁTICOS } \\
\text { ECONÓMICOS }\end{array}$} & GRI 201: Desempeño económico \\
\hline & GRI 202: Presencia en el mercado \\
\hline & GRI 203: Impactos económicos indirectos \\
\hline & GRI 204: Prácticas de adquisición \\
\hline & GRI 205: Anticorrupción \\
\hline & GRI 206: Competencia desleal \\
\hline \multirow{8}{*}{$\begin{array}{l}\text { GRI 300: ESTÁNDARES TEMÁTICOS } \\
\text { AMBIENTALES }\end{array}$} & GRI 301: Materiales \\
\hline & GRI 302: Energía \\
\hline & GRI 303: Agua \\
\hline & GRI 304: Biodiversidad \\
\hline & GRI 305: Emisiones \\
\hline & GRI 306: Efluentes y residuos \\
\hline & GRI 307: Cumplimiento ambiental \\
\hline & GRI 308: Evaluación ambiental de proveedores \\
\hline \multirow{19}{*}{$\begin{array}{c}\text { GRI 400: ESTÁNDARES TEMÁTICOS } \\
\text { SOCIALES }\end{array}$} & GRI 401: Empleo \\
\hline & GRI 402: Relaciones trabajador-empresa \\
\hline & GRI 403: Salud y seguridad en el trabajo \\
\hline & GRI 404: Formación y enseñanza \\
\hline & GRI 405: Diversidad e igualdad de oportunidades \\
\hline & GRI 406: No discriminación \\
\hline & GRI 407: Libertad de asociación y negociación colectiva \\
\hline & GRI 408: Trabajo infantil \\
\hline & GRI 409: Trabajo forzoso u obligatorio \\
\hline & GRI 410: Prácticas en materia de seguridad \\
\hline & GRI 411: Derechos de los pueblos indígenas \\
\hline & GRI 412: Evaluación de derechos humanos \\
\hline & GRI 413: Comunidades locales \\
\hline & GRI 414: Evaluación social de los proveedores \\
\hline & GRI 415: Política pública \\
\hline & GRI 416: Salud y seguridad de los clientes \\
\hline & GRI 417: Marketing y etiquetado \\
\hline & GRI 418: Privacidad del cliente \\
\hline & GRI 419: Cumplimiento socioeconómico \\
\hline
\end{tabular}

Elaboración propia a partir de www.globalreporting.org/standards/

Entre los objetivos de generar las memorias de sostenibilidad está el de reflejar el impacto de los programas de RSE en los resultados económicos de la empresa. Para ello, la ciencia contable "se ha visto en la necesidad de reorientar sus metodologías de medición, valoración y revelación de los hechos y transacciones ejecutadas por las 
organizaciones" (Hernández, Escobar, 2017: 49). De igual manera, se debe resaltar que la contabilidad ya no solo se limita a presentar información de índole únicamente monetaria, sino también no monetaria.

Establecer los usuarios de la información es reconocer que la contabilidad no solo debe centrarse en la información que entrega en los estados financieros, sino que se hace responsable de informar sobre cómo se produce y se distribuye la riqueza a través de la información de carácter monetaria y no monetaria. (Jiménez, 2012: 227-228)
Es importante señalar que en los últimos años los informes de sostenibilidad han dejado de ser únicamente fuente de información de la parte social y medioambiental de la gestión que ejecutan las empresas. Más bien se han convertido en un medio de comunicación sobre el capital intelectual de aspectos como la filosofía, la visión institucional; también la formación y desarrollo del capital humano, que se da a conocer a sus grupos de interés o stakeholders (Tejedo, 2016: 108). Entre los indicadores que se deben considerar en el informe de capital intelectual estarían:

Figura 3. Indicadores para medir intangibles

\begin{tabular}{|l|}
\hline \multicolumn{1}{|c|}{ Capital humano } \\
\hline - Personal altamente cualificado \\
- Actividades de formación \\
- Satisfacción de empleados \\
- Rotación de puestos de trabajo \\
- Flexibilidad del capital \\
humano \\
\hline
\end{tabular}

\begin{tabular}{|l|}
\hline \multicolumn{1}{|c|}{ Capital estructural } \\
\hline - Patentes \\
- Actividades de I+D \\
- Aumento de los \\
procesos regulados \\
- Flexibilidad del capital \\
estructural \\
\hline
\end{tabular}

\begin{tabular}{|l|}
\hline \multicolumn{1}{|c|}{ Capital relacional } \\
\hline - Satisfacción de clientes \\
- Lealtad de consumidores \\
- Marketing directo \\
\\
\hline
\end{tabular}

Elaboración propia a partir de Cañibano (2001)

Asimismo, se puede decir que ser socialmente responsable como empresa aporta, sin duda, al Cl. Su impacto se puede observar en la siguiente ilustración que re- presenta los resultados de la comprobación de varias hipótesis con respecto al efecto de la responsabilidad social corporativa frente a cada uno de los componentes del $\mathrm{Cl}$ :

Figura 4. Resultado del contraste de hipótesis

\begin{tabular}{|l|l|c|c|}
\hline Variable independiente & \multicolumn{1}{|c|}{ Hipótesis } & Efecto esperado & Verificación \\
\hline $\begin{array}{l}\text { Responsabilidad Social Cor- } \\
\text { porativa (RSC) }\end{array}$ & $\begin{array}{l}\text { H1:La responsabilidad social corporativa tiene } \\
\text { un impacto positivo y significativo en la for- } \\
\text { mación de capital humano }\end{array}$ & Signo positivo & Sí \\
\hline $\begin{array}{l}\text { Responsabilidad Social Cor- } \\
\text { porativa (RSC) }\end{array}$ & $\begin{array}{l}\text { H2:La responsabilidad social corporativa tie- } \\
\text { ne un impacto positivo y significativo en la } \\
\text { formación de capital de innovación }\end{array}$ & Signo positivo & Sí \\
\hline $\begin{array}{l}\text { Responsabilidad Social Cor- } \\
\text { porativa (RSC) }\end{array}$ & $\begin{array}{l}\text { H3:La responsabilidad social corporativa tie- } \\
\text { ne un impacto positivo y significativo en la } \\
\text { formación de capital de procesos. }\end{array}$ & Signo positivo & Sí \\
\hline $\begin{array}{l}\text { Responsabilidad Social Cor- } \\
\text { porativa (RSC) }\end{array}$ & $\begin{array}{l}\text { H4:La responsabilidad social corporativa tie- } \\
\text { ne unpacto positivo y significativo en la } \\
\text { formación de capital social o relacional. }\end{array}$ & Signo positivo & Sí \\
\hline
\end{tabular}

Fuente: Stanimirov Stoychev (2014) 
A partir de la ilustración 4 se comprende que a través de los programas de RSE se puede generar un nuevo conocimiento de cómo mejorar procesos que, a su vez, permitan crear un nuevo valor agregado para las empresas mediante el uso de herramientas tecnológicas así como nuevas competencias en su recurso humano que, en suma, se convertirá en un nuevo capital intelectual y que dichos resultados se deben plasmar en informes que pretenden comunicar a sus grupos de interés sobre la gestión realizada encontrando algunas similitudes entre ambos reportes.

Se destaca claramente que en ambos casos se busca lograr una mayor transparencia con todos los que se relacionan con la empresa, los llamados "stakeholders": accionistas, empleados, clientes, gobierno, opinión pública. Relacionando esto con

\section{El Informe Global Intangible}

No cabe duda de que contar con informes de la situación económica y financiera de una empresa en estos momentos no es suficiente para que los administradores tomen las mejores decisiones. De ahí que disponer de información sobre los intangibles de las empresas contribuye a hacerse un panorama más general de una compañía frente a sus grupos de interés. Para esto, se han mencionado anteriormente los informes de sostenibilidad y de capital intelectual; sin embargo, al tener los dos informes con información en común se está hablando ya de una fusión de ambos reportes denominado Informe Global Intangible (IGR, por sus siglas en inglés) o The Intangible Global Report.

Castilla-Polo y Gallardo-Vázquez (2008) justifican teóricamente la existencia de importantes áreas en común entre los Estados de Capital Intelectual y las Memorias de Sostenibilidad, proponiendo la incorporación de los temas sociales al primero de los documentos, el Estado de Capital Intelectual. Para Veltri y Nardo (2013) el concepto de capital intelectual, lo que estos informes buscan es aumentar el llamado capital relacional. Tanto un informe como el otro son un complemento importante a la tradicional información económico-financiera que producen las empresas y organizaciones. (Horta, 2004: 18)

En relación con lo anterior, el objetivo principal de ambos reportes tanto del Informe de RSE o Balance Social como del Informe de Capital Intelectual, es mantener informados y transmitir confianza a sus grupos de interés o stakeholders, transparentando la información tangible como intangible que se genera dentro de las empresas, convirtiendo estos informes en un complemento de suma importancia a la hora de tomar decisiones además de los balances tradicionales que la contabilidad nos tiene acostumbrados a generar.

ambos informes proporcionan información sobre los intangibles de una empresa, de ahí su similitud y la posibilidad de elaborar un único informe denominado The Intangible Global Report IGR. Se decantan por combinar ambas informaciones en un tipo de documento genérico llamado IGR. (Citado en Castilla-Polo, Gallardo-Vázquez, 2015: pp. 8-9)

Ante este panorama, la consultora independiente Brand Finance presenta cada año un informe anual del valor de los intangibles en el que se analiza cómo fluctúa el valor de los activos intangibles en los mercados bursátiles en todo el mundo. En su edición en español de octubre de 2018, junto con la colaboración de Corporate Excellence, indica:

El Global Intangible Finance Tracker (GIFTTM) de Brand Finance desvela que el $52 \%$ del valor global de todas las empresas que mundialmente cotizan en bolsa reside en los intangibles, cuyo valor total ha alcanzado los 57,3 billones de dólares -una cifra que nunca antes se había alcanzado-. Sin embargo, y tal y como señalan los resulta- 
dos de este estudio, es preocupante que la gran mayoría de esos activos intangibles permanezcan sin contabilizar en los balances. (Brand Finance, 2018: 24)

De lo anterior, es fundamental destacar que el no presentar los activos intangibles en los balances contables se ha convertido en una tendencia mundial, tomando en cuenta que el estudio al que hace referencia la cita anterior es realizada a las marcas más grandes del mundo. El principal inconveniente está en que desde el punto de vista contable y bajo las Normas Internacionales de Información Financiera (NIIF), también conocidas como International Financial Reporting Standards (IFRS, por sus siglas en inglés), "solo se reconoce el valor de los intangibles adquiridos a terceros y no los generados internamente como consecuencia de la buena gestión organizativa" (Brand Finance, 2018: 8).

A estos activos generados de manera interna se les denomina "intangibles ocultos", y que al no ser identificables, tal como establece la norma contable, no pueden ser contabilizados como cualquier otra transacción contable.

En dicho estudio se destaca que la variación del valor oculto de las empresas, que analiza la consultora Brand Finance, muestra un mayor crecimiento de un año a otro; es decir: de $25 \%$, y la tendencia para el futuro es que se siga incrementando.

En la actualidad no basta con tener los tradicionales balances financieros, pues, como ya se analizó, existen otros tipos de reportes como los informes de sostenibilidad,

\section{Conclusiones}

La contabilidad, vista desde su dimensión social, no solo se centra en proporcionar información financiera, sino en exponer información de los aspectos sociales y medioambientales de los que la empresa aspira a generar nuevas ventajas competitivas. Para que se producen a través de los programas de RSE, o los informes de capital intelectual, los cuales abarcan los recursos intangibles. También está la fusión de ambos informes a través del llamado Informe Global Intangible. La realidad, no obstante, es que falta mucho camino por recorrer en cuanto a la medición, evaluación y registro contable de los intangibles que se elaboran o crean en una empresa. Para estos fines es importante que los gerentes, administradores, propietarios, accionistas de empresa se involucren en el proceso, informándose sobre estos temas, además de ofrecer el apoyo y la capacitación a su talento humano con respecto a la normativa contable, financiera y tributaria local e internacional que se está empleando en el mundo empresarial y que no puede pasar inadvertida.

Evidentemente, la responsabilidad social empresarial y el capital intelectual en las empresas se vienen abordando desde hace algunos años. Tanto a escala regional como mundial todavía existen aspectos puntuales que se deben tratar y profundizar, tal es el caso de los reportes, los indicadores y la forma de contabilizar dichos resultados para que se vean reflejados en nuevos reportes, además de los informes tradicionales. También la empresa debe dar lugar a procesos éticos que apunten a ser responsables con los empleados, la sociedad y el medioambiente; todos trabajando como una sola red de conocimiento que contribuya a un desarrollo sostenible para el bien de las actuales y futuras generaciones.

esto los profesionales del área deben asumir el reto de presentar reportes con indicadores para medir y evaluar los resultados de los planes o programas implementados con miras hacia la RSE a través de las memorias de sostenibilidad. 
No cabe duda de que un modelo de gestión del conocimiento, basado en valores morales y éticos, así como en preceptos de responsabilidad social, ayudará a las empresas a conseguir un valor agregado que les permita diferenciarse de la competencia.

Es necesario que se cree una red de conocimiento en la que confluyan el Estado, la empresa y la universidad como un engranaje para la elaboración de un modelo de gestión del conocimiento, que se fundamente en preceptos de responsabilidad social. Esto, con el propósito de obtener proyectos o productos que puedan ser patentados y distribuidos

\section{Referencias}

Angulo, R. (2017). Gestión del conocimiento y aprendizaje organizacional: una visión integral. Informes Psicológicos, 17(1), 53-70.

Barabraham, R. S. S. (2017). Balance social y contabilidad social: lineamientos para un modelo de gestión. Herramientas las de auditoría interna y externa para una visión prospectiva. Recuperado de https://rdu. unc.edu.ar/handle/11086/5616

Brand Finance (2018). Informe anual del valor de los intangibles en el mundo. Recuperado de https://brandfinance.com/images/uplo ad/gift_esp.pdf

Bravo, A.; Alfonzo, E. (2015). Gestión del conocimiento y medición del capital intelectual como recurso intangible en las organizaciones que aprenden. Análisis comparativo desde sus modelos originarios. Cicag, 13(1), 223-234.

Cañibano, L. (2001). La relevancia de los intangibles en el análisis de la situación financiera de la empresa. IVIE, Valencia, 1-18.

Caridad, M.; Hernández, P.; Pelekais, C. de. (2014). Responsabilidad gerencial: elemento integrador de la sustentabilidad en la responsabilidad social empresarial. Opción, 30(75), 35-54.

Castilla-Polo, F.; Gallardo-Vázquez, D. (2015). Responsabilidad social corporativa y capital intelectual en las organizaciones. En Congreso AECA. como aporte para el mejoramiento de los procesos de medición y valoración de resultados de proyectos de RSE o de la información intangible que posee una organización.

A su vez, es necesario que para generar capital intelectual, se invierta en el recurso humano al que muchas veces, sin embargo, los altos directivos olvidan, pues lo ven únicamente como un gasto. Para este fin se deben concebir políticas institucionales que se orienten hacia la investigación, la creatividad, el desarrollo de las competencias, habilidades, y motivándolo también hacia el crecimiento personal y profesional.

Cegarra, J. G.; Martínez, A. (2017). Gestión del conocimiento: una ventaja competitiva. ESIC Editorial.

Fernández Lorenzo, Liliana E, \& Geba, Norma E. (2005). Contabilidad Social y Gestión en ONGs. Revista Venezolana de Gerencia, 10(32), 545-563. Recuperado en 28 de mayode2019, dehttp://www.scielo.org.ve/ scielo.php?script=sci_arttext $\&$ pid=S131599842005000400002\&lng=es\&tlng=es.

Hernández, D.; Escobar, A. (2017). Modelo de contabilidad social como herramienta de gestión para la responsabilidad social empresarial. International Journal of Management Science and Operations Research, 2(1), 44-56.

Horta, R. (2004). Informes de capital intelectual y de responsabilidad social corporativa. Análisis crítico. Electrónica FCE.

Jiménez, R. (2012). Contabilidad: entre la responsabilidad social y el interés público. Criterio Libre, 10(17), 219-234. Recuperado de https://doi.org/10.18041/1900-0642/criteriolibre.17.1149

Madrigal, B. E. (2009). Capital humano e intelectual: su evaluación. Observatorio Laboral Revista Venezolana, 2(3), 65-81.

Ortiz de Urbina Criado, M. (2003). Mediación y auditoría del capital intelectual. El profesional de la información, 12(4), 282-289. 
Pardo, M.; Armas, R.; Chamba, L. (2017). Valoración del capital intelectual y su impacto en la rentabilidad financiera en empresas del sector industrial del Ecuador. Publicando, 4(13), 193-206.

Ruiz, M. de los A.; Font, E.; Lazcano, C. (2016). El impacto de los intangibles en la economía del conocimiento. Economía y Desarrollo, 155(2). Recuperado de http://www.econdesarrollo.uh.cu/index.php/RED/article/ view/80

Stanimirov Stoychev, I. (2014). Capital intelectual y responsabilidad social corporativa: una investigación empírica.
Solíz, N. A.; Mena, V.; Lara, T. (2017). Cultura y gestión del conocimiento en organizaciones del Ecuador. Publicando, 4(11 (2)), 70-83.

Tejedo, F. (2016). Información de los recursos intangibles ocultos: ¿memorias de sostenibilidad o informe anual? European Research on Management and Business Economics, 22(2), 101-109. Recuperado de https://doi. org/10.1016/j.iedee.2015.06.001

Vaca, R. M.; Moreno, M. J.; Riquel, F. J. (2007). Análisis de la responsabilidad social corporativa desde tres enfoques: stakeholders, capital intelectual y teoría institucional.

Conocimiento, innovación y emprendedores: camino al futuro, 222. Universidad de La Rioja. 